\title{
Il «tumore» endorbitario del Feldmaresciallo Radetzky guarito «dalla terapia omeopatica»
}

\author{
di Luigi Belloni
}

Attorno al 1840 il Feldmaresciallo Johann Joseph Franz Karl Wenzel Graf Radetzky von Radetz (1766-1858) fu colpito da un «tumore» endorbitario destro inglobante buona parte del bulbo oculare, che guarì completamente in seguito alla terapia omeopatica praticatagli dal suo medico di fiducia Christoph Hartung (1779-1857) ${ }^{1}$, «dirigirender Stabsfeldarzt» nell'armata d'Italia e convinto seguace della omeopatia. Dopo aver seguito il Feldmaresciallo per l'intero calvario, Hartung lasciò ai posteri la storia clinica sulle colonne di un periodico di medicina omeopatica ${ }^{2}$. Detta storia si compone di cinque successivi rapporti, tutti datati 1841, e precisamente: 1) 7 Gennaio; 2) 19 Febbraio; 3) 16 Marzo; 4) 22 Aprile; 5) 12 Giugno.

Estraggo ora dalla relazione di Hartung quanto è sufficiente per seguire le vicende morbose del Feldmaresciallo. Questi aveva ormai largamente superato i settant'anni, dopo lunghi decenni di oneroso servizio, mantenendosi in condizioni mentali e fisiche eccezionalmente floride e conservando una capacità di lavoro dal primo mattino alla sera. Da anni era soltanto affetto da tosse da irritazione tracheale, cui non faceva peraltro gran caso. Negli ultimi anni, fino al 1836, comparve, a ciascun autunno, una febbre catarrale infiammatoria, che regolarmente scompariva con pochi giorni di riposo e qualche cura medica.

Nel 1836 una disgraziata caduta sul bordo del letto gli provocò una frattura affondata della $6 .^{\mathrm{a}}$ e $7 .^{\mathrm{a}}$ costa complicata da una pericolosa polmonite, guarita peraltro con terapia medica in $7 .^{a}$ giornata, allorchè il paziente espettorò 10-12 oncie di pus fetido. Da allora, pur perdurando la tosse abituale, cessò la febbre catarrale infiammatoria.

Buoni, salvo qualche indisposizione, gli anni 1837 e 1838: soltanto si presentavano saltuariamente, come già prima, cefalea frontale, eruttazioni acide e diarrea senza ulteriori sviluppi morbosi, dato che questi disturbi erano tosto eliminati con appropriati medicamenti.

Nel bimestre Luglio-Agosto 1839 il Feldmaresciallo sofferse di congestioni alla testa, per lo più accompagnate da violente vertigini fino allo svenimento. Anche questi disturbi scomparvero allorchè Radetzky lasciò 
Milano per recarsi al campo a Pordenone; ma subito dopo avvertì senso di pressione all'occhio destro, con infiammazione palpebrale, lacrimazione, saltuaria protrusione del bulbo oculare, senso di pressione alla fronte. I disturbi erano sempre alleviati con mezzi idonei; soltanto la palpebra inferiore rimaneva infiammata. Così, fra miglioramenti e peggioramenti, arrivò la primavera del 1840. Nel mese di Maggio, unico, ma violente, attacco di vertigine. Nel trimestre Luglio-Settembre 1840, Radetzky partecipò nuovamente al campo, e in buone condizioni, salvo lacrimazione continua dell'occhio destro.

Il 9 Ottobre, durante le grosse manovre nella zona di Castiglione delle Stiviere, rimase in sella per cinque ore esponendosi alternativamente al calore dei fondovalle e all'aria fresca delle alture. Poco dopo comparve arrossamento del volto. Alla sera insorse febbre elevata con dolore di tale violenza alla fronte destra, che il paziente, pur non aduso a lamentarsi, definiva insopportabile. L'occhio era fortemente infiammato e alquanto sporgente fuori dall'orbita: polso pieno, duro, apoplettico. Hartung somministrò i mezzi necessari secondo la sua scienza. All'ora 1 di notte la cefalea si attenuò e l'occhio rientrò. All'indomani il Feldmaresciallo passò in rivista le truppe rimanendo in sella per ben sei ore. Alla sera stava bene: era soltanto un po' stanco. Dopo un giorno di riposo, viaggio di rientro a Milano: l'occhio destro era ancora arrossato e lacrimante.

A Milano, notevole miglioramento: dopo un trattamento protratto persistono soltanto arrossamento alla palpebra inferiore, lacrimazione e un tumore all'angolo esterno dell'occhio verso l'alto nell'orbita, peraltro senza dolore, nè compromissione del visus.

Verso la fine di Ottobre Radetzky si trasferì per sei settimane a Verona. In pieno maltempo si recò da Verona a Modena: e qui si formò alla palpebra inferiore un tumore delle dimensioni di un fagiolo, procedente dall'angolo interno dell'occhio; il preesistente tumore all'angolo esterno aumentò di volume; notevole sporgenza dell'occhio fuori dalla sua cavità; dolori intermittenti al seno frontale e violente congestioni alla testa.

Dopo otto giorni Radetzky rientrò a Milano, dove Hartung osservò il reperto testè descritto. Le condizioni del Feldmaresciallo apparvero preoccupanti al suo medico grazie al preciso rilievo che nell'orbita si era formata una spugna ${ }^{3}$, la cui proliferazione, afferma Hartung, è temibile. Estinta con cataplasmi emollienti la infiammazione che interessava la guancia destra nella sua totalità, Hartung si propose di contrastare, se possibile, la proliferazione della spugna per mantenere in vita il paziente, data la 
frequente minaccia di attacchi apoplettici. Il trattamento omeopatico praticato da Hartung e descritto nella sua relazione, permise a Radetzky di mantenersi in ottimo stato generale, salvo frequenti congestioni di testa; ma la proliferazione del tumore spugnoso non subì alcun arresto.

Il già descritto tumore sopra l'angolo esterno dell'occhio, reso meglio visibile dal suo colore azzurro-rossiccio, era anzi aumentato di volume: e così pure, il tumore procedente dall'angolo interno dell'occhio. Fra il bulbo oculare e la palpebra inferiore era per sovrappiù comparso un altro tumore spugnoso, elastico, granuloso, rosso pallido, indolente. A causa di queste lesioni, il bulbo era non soltanto spinto fuori dall'orbita, ma anche deviato rispetto al normale asse ottico: la pupilla era diretta verso l'alto e l'esterno, e la libera mobilità del bulbo era limitata, senza alcuna compromissione del visus, tant'è vero che ancora il giorno precedente Radetzky aveva scritto una lettera e sbrigato altre faccende senza occhiali.

Desideroso del parere di un altro medico, Hartung ottenne che venisse chiamato a consulto il Prof. Francesco Flarer (1791-1859) ${ }^{4}$, clinico oculista all'Università di Pavia. Al termine del consulto, svoltosi il 6 Gennaio 1841, Flarer confermò la diagnosi e la prognosi di Hartung, ulteriormente aggravandola, al punto da affermare: «Non possiamo far nulla». Ciò nonostante Hartung fece osservare l'opportunità di una prescrizione per sollevare lo spirito del paziente: fu decisa la somministrazione di $1 / 4$ di grano di Mercurio subl. corr. pro die.

Così termina il $1^{\circ}$ rapporto Hartung, datato 7 Gennaio 1841. Seguì, il 19 Febbraio, il $2^{\circ}$ rapporto, ricco di ulteriori dettagli sul consulto col Flarer.

Il Flarer, dunque, dichiarò a Hartung e ad altri che, nel caso in esame, la guarigione non era possibile nè con l'allopatia, nè con l'omeopatia, nè con l'idropatia, nè con qualsiasi altro metodo: Radetzky sarebbe inevitabilmente morto per cachessia o per apoplessia. Il prof. Flarer non poteva ordinare nulla: soltanto dietro invito del curante, e per sollevare il morale del paziente, prescrisse, in presenza di costui sublimato corrosivo alla dose di $1 / 4$ di grano pro die, come si è detto nella precedente relazione. Flarer era pienamente convinto della inutilità di questo farmaco; ma dichiarò al curante di non conoscere «alcun mezzo più idoneo». Hartung seguì il consiglio di Flarer, pur riducendo a un terzo la dose ( $1 / 12$ di grano); ma nemmeno questa dose era tollerata dal paziente, perchè fonte di violentissime e minacciose congestioni alla testa, che richiedevano la somministrazione di antidoti (non specificati!). 
Proprio in questo periodo Hartung stese una relazione clinica, e la inoltrò a Vienna tramite Radetzky, dato che, in ragione del suo ufficio, considerava la salute del Feldmaresciallo quale suo compito più sacro.

La relazione fu letta da principi e da arciduchi, nonchè dallo stesso imperatore, il quale dispose l'invio a Milano del Dr. Friedrich Jäger von Jaxtthal (1784-1871) ${ }^{5}$, Stabsarzt e Professore di Clinica oculistica alla I. R. Accademia Medico-chirurgica Giuseppina di Vienna, affinchè si consultasse con Hartung e decidessero assieme di mettere in atto ogni mezzo possibile per salvare la vita del Feldmaresciallo.

Nel frattempo il curante cercò di mantenere l'organismo in buone condizioni generali, pur tenendo conto dell'accrescimento della spugna, alla quale non riusciva a porre alcun freno. La spugna aumentò così rapidamente, che formò all'angolo esterno dell'occhio, nella regione della ghiandola lacrimale, una eminenza dura, a cercine, grigio-azzurra, che superava le 8 linee di larghezza: e questa eminenza si estendeva, con decorso arcuato, lungo la palpebra superiore, gradualmente assottigliandosi e appiattendosi verso l'angolo interno dell'occhio. Da questo angolo poi si presentava anche sotto la palpebra inferiore, un tumore grigio-azzurro, che formava una parete perpendicolare verso l'occhio e si perdeva declinando verso il naso. Il tumore delle dimensioni di fagiolo all'angolo interno dell'occhio si era triplicato in grandezza, presentava un colore rosso più carico, ed era divenuto più irregolare $\mathrm{e}$ più duro. Il tumore rosso pallido che protrudeva fra il bulbo $\mathrm{e}$ la palpebra inferiore, sporgeva liberamente fino alla lunghezza di $1 / 3$ di pollice, e lasciava evidentemente riconoscere la natura spugnosa del prodotto morboso formantesi lungo tutta la circonferenza del bulbo oculare, mentre lo stesso tumore sopra l'angolo esterno dell'occhio era soltanto accennato attraverso il colore grigio-azzurro della cute che lo copriva e non era direttamente visibile. Nell'intera formazione spugnosa si manifestavano dolori di diverso tipo: punture, strappi, bruciore, prurito; e si producevano facilmente emorragie. L'occhio stesso era però indolente e trovavasi come in un anfratto fra queste due prominenze; era al tempo stesso protruso dall'orbita, e come già in precedenza, rivolto verso l'alto e verso l'angolo esterno dell'occhio, ma era assolutamente immobile; anche il visus era contemporaneamente alterato: infatti, verso il lato esterno il paziente vedeva ogni oggetto come un corpo nero, senza riuscire a distinguere cosa fosse. La congiuntiva era rilassata (chemosi) e di color rosso scuro con riflessi bluastri. Al mattino, la fessura palpebrale era conglutinata da muco biancastro, purulento e viscoso; mentre durante la giornata aumentavano la 
irritabilità dell'occhio verso la luce, e la lacrimazione; alla sera subentravano maggior calore, secchezza e aumento del dolore.

In queste condizioni trovavasi la malattia di Radetzky il giorno 25 Gennaio, allorchè arrivò a Milano il prof. Jäger. Tosto convocato il prof. Flarer, il consulto a tre ebbe luogo il 26 a mezzogiorno.

Il prof. Jäger dichiarò la malattia inguaribile, poichè essa consisteva in una discrasia ${ }^{6}$, talchè l'intervento chirurgico non poteva portare nessun aiuto; d'altronde egli non conosceva alcun rimedio per uso interno, dato che finora questa malattia non era stata mai guarita.

Il prof. Flarer tornò a ripetere quanto aveva già detto nel consulto precedente.

In base al punto di vista della scuola razionale e alla sua quarantennale esperienza, Hartung dovette dichiararsi d'accordo col prof. Jäger.

A questo punto era difficile, sia pure con tutto il tatto, far capire al paziente la vera prognosi senza turbarlo e senza fargli perdere la fede nel curante. Egli venne pertanto assicurato che i due consulenti confermavano la loro piena fiducia in Hartung e nei suoi rimedi. Col tempo sarebbero subentrate emorragie, dolori, ecc., e il tumore sarebbe passato a suppurazione, nel qual caso il curante avrebbe provveduto ad applicare i rimedi necessari.

Il prof. Jäger comunicò anche al Vicerè l'esito del consulto, e dopo 4 giorni di permanenza a Milano si congedò cordialmente dal curante e ripartì per Vienna. Anche il Vicerè ripetè a Radetzky la piena fiducia che i due consulenti riponevano nel curante e nei suoi rimedi.

Nella visita successiva, Radetzky abbracciò Hartung e gli fece questa dichiarazione: «Amico! ora se ne sono andati tutti: io sono nelle vostre mani: fate di me ciò che volete: ho piena fiducia in voi: non voglio più vedere altro medico».

Hartung fu profondamente commosso da queste parole: due dotti ed esperti medici e professori avevano dichiarato con apodittica certezza la inguaribilità della malattia; ed egli aveva dovuto approvare questo giudizio [...]. D'accordo, Jäger e Flarer erano medici razionali espertissimi che rifuggivano dalla omeopatia; ma proprio in base ai principi di questa Hartung aveva già guarito molti pazienti abbandonati come inguaribili dalla Scuola razionale. Pertanto egli continuò imperterrito e con tranquilla coscienza la via iniziata, finchè ottenne un innegabile successo: la cessazione della emorragia già iniziata; la scomparsa della minaccia di degenerazione cancerosa; la scomparsa completa dei dolori; la riduzione del tumore superiore sopra la ghiandola lacrimale a un modesto rilievo. 

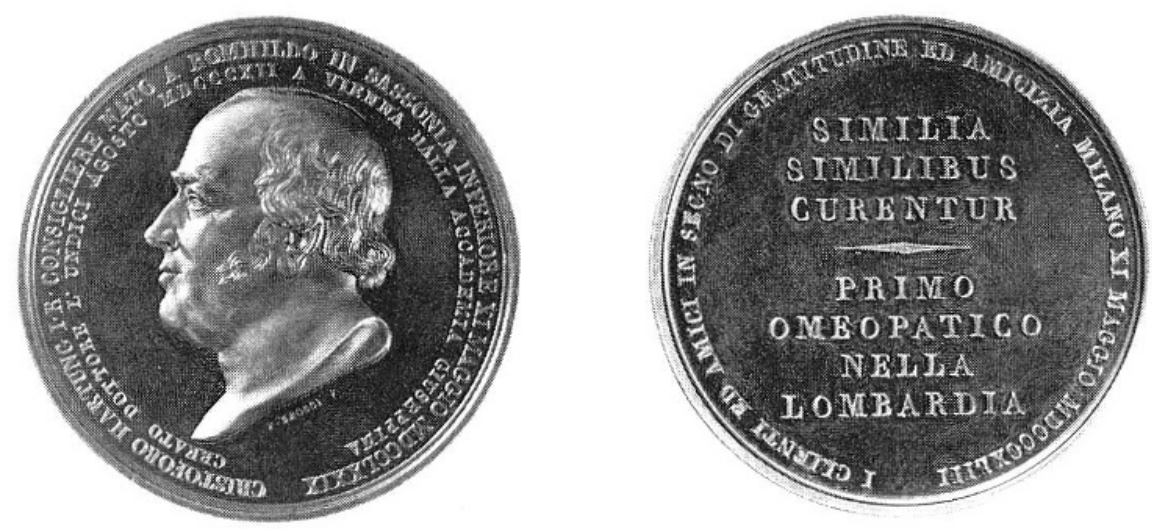

Figg. 1 e 2 - Diritto e rovescio della medaglia-ritratto offerta a Christoph Hartung nel 1843 dai Milanesi, fra i quali si era diffusa, in seguito alla guarigione di Radetzky, la sua fama di medico omeopatico. Diametro $41 \mathrm{~mm}$

Diritto - In giro, su due righe: CRISTOFORO HARTUNG I. R. CONSIGLIERE NATO A ROMHILDO IN SASSONIA INFERIORE XI MAGGIO MDCCLXXIX/CREATO DOTTORE L'UNDIGI AGOSTO MDGGCXII A VIENNA DALLA ACGADEMia giUSEPPINA. - Nel campo: testa di profilo a sinistra; sul taglio della spalla: F. BROGGI. F.

Rovescio - In giro: I CLIENTI ED AMICI IN SEGNO DI GRATITUDINE ED AMICIZIA MILANO XI maggio mdCCCXliII. - Nel campo: similia similibus curentur - Primo omeopatico Nella LOMBARDIA

Il margine superiore dell'orbita si presentava abbastanza libero; il tumore duro all'angolo interno dell'occhio, e così pure il tumore teso, o spugna, visibile fra il bulbo e la palpebra inferiore, si erano ridotti in tutte le direzioni, tanto che è ritornata la visibilità verso il naso e il paziente sa distinguere i singoli oggetti come nel precedente stato di salute. Il bulbo ha riacquistato la sua libera mobilità nell'orbita, e il colore naturale al pari di tutte le parti circostanti. Stato generale buono; spirito pieno d'entusiasmo; corpo attivo e in forze. Hartung continua il trattamento omeopatico, nella speranza, se non proprio di guarire il paziente, di mantenere i risultati raggiunti, dato che, soprattutto in un corpo a settant'anni largamente superati, è ben nota la malignità di questa malattia, di cui non si conoscevano fino allora casi guariti.

Così termina il $2^{\circ}$ rapporto, in data 19 Febbraio. Segue il $3^{\circ}$, breve, in data 16 Marzo.

Il miglioramento continua: persiste unicamente un piccolo tumore spugnoso, che è visibile soltanto scostando la palpebra inferiore. Stato generale ottimo. 
Al momento, Hartung rivela soltanto la posologia, non però la natura, dei due farmaci alternativamente somministrati, e segnala espressamente:

«Dopo ogni singola somministrazione, il paziente provava una sensazione, peraltro indolora e transitoria, di tutti i mali precedentemente sofferti, come un 〈alito〉 (〈Hauch〉), al quale subentrava l'azione guaritrice». Col che, il rude soldato, la solida colonna di Sua Maestà Imperiale e Reale Austriaca (S.M.I.R.A.), appare squisitamente sensibile alle finezze del «credo» omeopatico.

Nel $4^{\circ}$ rapporto (22 Aprile), il tumore della palpebra inferiore è quasi completamente scomparso: si osserva soltanto lieve ectropion, d'altronde frequente nei vecchi. L'occhio destro è ritornato perfettamente identico all'altro, e ha riacquistato pienamente il suo visus. Stato generale ottimo. Il Feldmaresciallo ha pienamente ripreso la normale attività. La malattia è ormai guarita; nè sono necessarie ulteriori relazioni.

Segue peraltro, in data 12 Giugno, un rapporto riassuntivo (il $5^{\circ}$ ). Hartung conferma la guarigione totale e si diffonde sugli effetti dei vari farmaci somministrati, o soltanto provati, ovviamente a dosi infinitesimali. Il rapporto è concluso dall'inno di vittoria: «Questa malattia dichiarata inguaribile, è stata invece guarita, in modo indoloro, rapido e duraturo, secondo i principi della omeopatia!». La fotografia a pagina 128-129 del Radetzky di Franz Herre (Milano 1982) non lascia riconoscere segni di asimmetria fra i due occhi, al pari di altre fotografie risalenti agli anni ' 50 del secolo scorso.

La vittoria della medicina alternativa, tanto più fulgida in ragione dell'importanza del paziente, valse a diffondere a Milano e in Lombardia la medicina omeopatica. Ne è testimone la medaglia coniata in onore di Hartung nel 1843 (Figg. 1 e 2).

La guarigione «omeopatica» del tumore endorbitario di Radetzky fornì anche lo spunto a polemiche ${ }^{7}$, che, iniziate in seguito alla pubblicazione della relazione di Hartung, si riaccenderanno soprattutto dopo le fulgide vittorie del Feldmaresciallo nella campagna d'Italia del 1848/9.

A questo punto, si pone il problema di individuare la natura del tumore endorbitario «dichiarato inguaribile» dai medici, e scomparso invece «in modo indoloro, rapido e duraturo» successivamente alla terapia omeopatica praticata da Hartung.

Un buon indizio verso la diagnosi ci è fornito dal «caso di aneurisma per anastomosi nell'orbita, guarito mediante legatura dell'arteria carotide 
comune» di Benjamin Travers (1783-1858) ${ }^{8}$. L'esauriente descrizione di Travers (donna di 34 anni) è accompagnata da perspicua iconografia, che viene qui riprodotta (Figg. 3 e 4) grazie alla affinità con taluni punti sostanziali della descrizione di Hartung. Nel suo complesso, essa può infatti servire a darci un'idea «mutatis mutandis» del «tumore» endorbitario di Radetzky. Le lesioni del caso Travers (vistose varici, più o meno nodulari, chemosi, tumefazione dei tessuti endorbitari e conseguente esoftalmo, etc.) (Fig. 3) sono tutte conseguenza del forte aumento di pressione trasmesso al sangue venoso dalla patologica comunicazione di questo col sangue arterioso. Ne è prova perspicua la scomparsa della sintomatologia in seguito alla legatura dell'arteria carotide comune al collo.
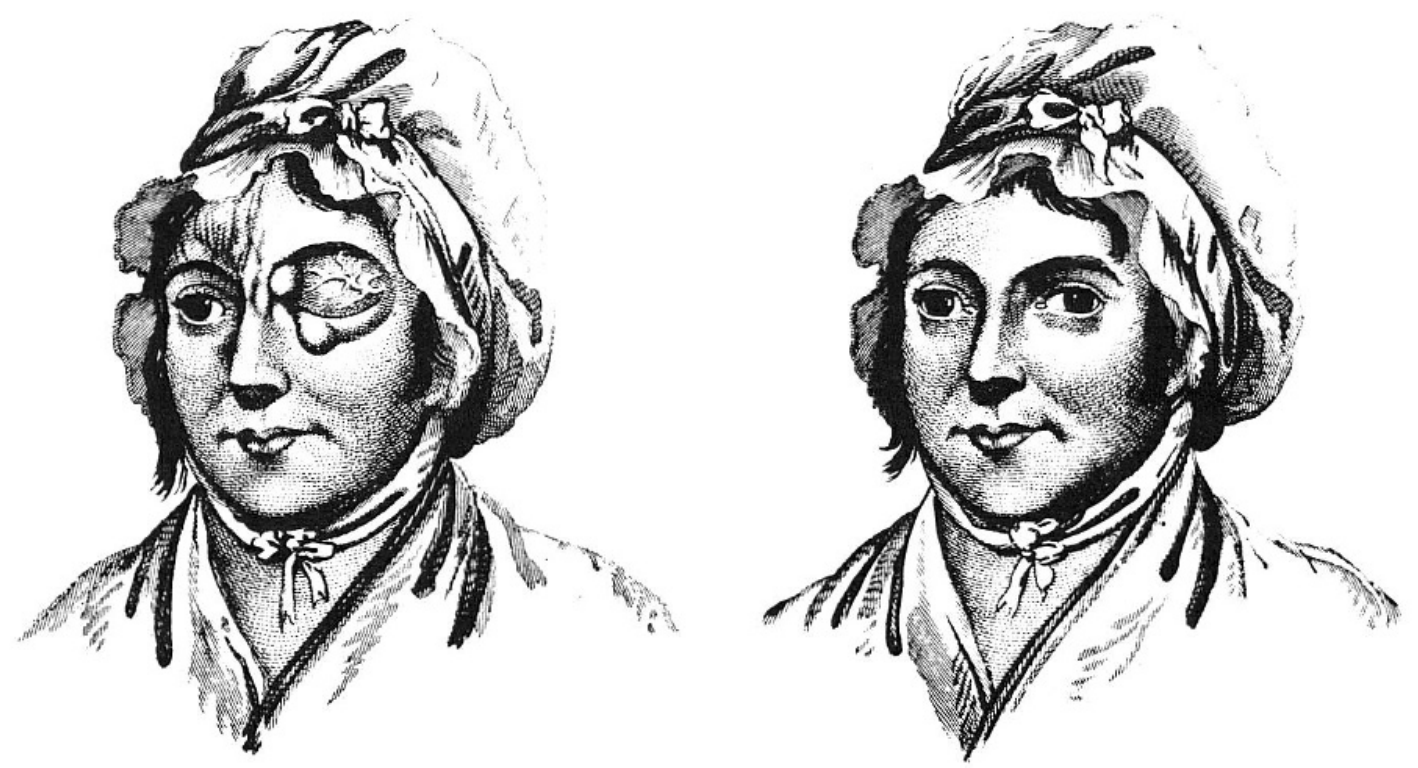

Figg. 3 e 4- La paziente di Benjamin Travers (donna di 34 anni) prima e dopo la legatura dell'arteria carotide comune sinistra. Le lesioni endorbitarie a sinistra si sovrappongono sostanzialmente, mutatis mutandis, a quelle del Feldmaresciallo Radetzky

Analogo al caso di Travers è quello descritto quattro anni più tardi da William Dalrymple (1772-1847) ${ }^{9}$, esso pure guarito mediante legatura dell'arteria carotide comune sinistra. Anche questo caso fu definito «aneurisma per anastomosi nell'orbita», ossia una condizione morbosa che assunse in seguito diversi nomi, fra cui «anastomosi artero-venosa»; «esoftalmo pulsante» (Sattler) ${ }^{10}$; «aneurisma carotido-cavernoso» (Dandy). ${ }^{11}$ 
Oggi viene preferito il termine «fistola carotido-cavernosa» (Hamby) ${ }^{12}$, afferente a una condizione anatomo-patologica individuata nell'ambiente di Auguste Nélaton (1807-73) ed egregiamente raffigurata, oltre che descritta, nella tesi di Delens ${ }^{13}$ (Fig. 5).

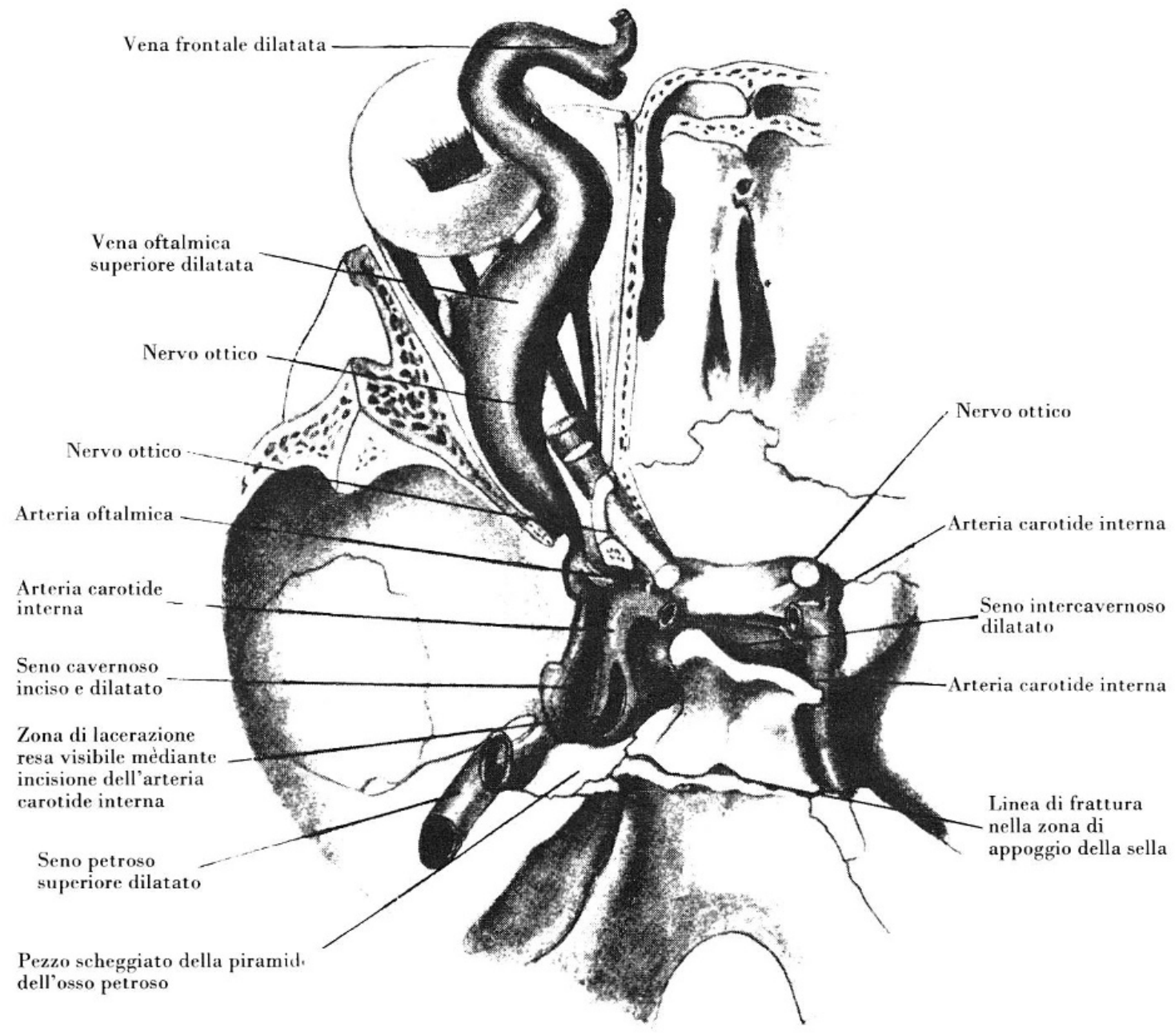

Fig. 5. - La base cranica di uno dei casi di Delens (1870) vista dal centro della cavità cranica (da Sattler)

Torna qui opportuno ricordare alcuni caratteri della fistola carotidocavernosa utili per un miglior chiarimento del caso Radetzky: 
1) L'insorgenza è spontanea nel $20-25 \%$ dei casi; altrimenti è riferibile a una etiologia traumatica. Potremmo desumere in proposito elementi, non tutti perspicui, della storia clinica; ma assai più fruttuoso potrebbe riuscire un esame del cranio, e soprattutto della base, in occasione di una eventuale ricognizione dei resti mortali del Feldmaresciallo nella cappella del Heldenberg ${ }^{14}$.

2) L'occhio protruso presenta abitualmente una pulsazione sincrona col polso del paziente, che può essere percepibile con la vista, ma molto più spesso apprezzabile col tatto. Tale pulsazione è completamente assente nel $5 \%$ circa dei casi. Nella storia clinica, Hartung non segnala pulsazioni nè visibili, nè palpabili; e nemmeno fa menzione di una palpazione del bulbo che molto probabilmente non fu nemmeno praticata.

3) Nel 5-10\% dei casi la guarigione insorge spontanea: e in alcuni casi così guariti fu anatomicamente costatata una trombosi globale del seno cavernoso e dello squarcio della carotide. Ne deriva la normalizzazione della pressione venosa con la conseguente regressione della protrusione del bulbo, del tumore endorbitario, e dell'esoftalmo.

La lesione sofferta da Radetzky può pertanto essere diagnosticata come una fistola carotido-cavernosa evoluta in guarigione spontanea.

L'errore diagnostico dei tre medici che curarono il Feldmaresciallo impedì il riconoscimento del carattere spontaneo della guarigione, la quale potè pertanto essere erroneamente attribuita alla cura omeopatica praticata da Hartung.

Note

${ }^{1}$ Rudolf Tischner, Geschichte der Homöopathie, Leipzig 1939, p. 774. Un ritratto miniato di Christoph Hartung è riprodotto nell'articolo: Hans Walter, Die homöopathische Hausapotheke des Feldmarschalls Radetzky, in «Zur Geschichte der Pharmazie», 19 (1967), p. 16.

${ }^{2}$ Hartung, Krankheits-Geschichte Sr. Excellenz des Herrn Feldmarschalls Grafen von Radetzky, in «Allgemeine Homöopathische Zeitung», vol.20, No 10 e $\mathrm{N}^{\circ} 11$ del 19 e 26 Luglio 1841, pp. 145-152 e 161-168.

${ }^{3}$ La nosografia dei tumori è molto confusa nel periodo anteriore alla Cellularpathologie (1858) di R.Virchow. La «spugna» menzionata da Hartung rientra nella artificiosa specie neoplastica detta «fungo ematode», nella quale confluivano sia l'«encefaloide ematode» corrispondente a un sarcoma ricco di sangue più o meno stravasato, che era considerato un tumore ad alta malignità, sia i tumori puramente sanguigni, ossia formazioni per lo più benigne in senso oncologico. 
${ }^{4}$ Francesco Flarer (1791-1859), nativo di Tirolo presso Merano, conseguì nel 1815 la laurea in medicina e la laurea in chirurgia all'Università di Pavia. Dietro interessamento di Antonio Scarpa (1752-1832), oculista insigne oltre che grande anatomo e chirurgo, il Flarer fu inviato a Vienna, perchè si perfezionasse alla scuola di Georg Joseph Beer (1763-1821), fondatore della locale Clinica Oculistica. Conseguito nel 1817 il diploma di maestro oculista («magister rei oculariae»), il Flarer vinse nel 1819 il concorso per la cattedra di Oculistica teorico-pratica, allora creata alla Università di Pavia. Cfr. Achille Monti, Francesco Flarer e gli Atesini all'Università di Pavia nella prima metà del secolo $X I X$, in «Archivio per l'Alto Adige», 26 (1931), pp. 187-255.

${ }^{5}$ L. de Wecker, Frédéric Jäger, Chevalier de Jaxthal, [!], in «Annales d'Oculistique», 69 ( = 9 d.S. 10), 1873, pp. 85-93; Georg Preyss, Das Leben und Wirken des k.k. Raths, Stabsfeldarzt und Professor der Augenheilkunde am Josefinum Dr. Friedrich Jäger Ritter v. Jaxtthal, Wien 1877; Erna Lesky, Die Wiener Medizinische Schule im 19. Jahrhundert (=Studien zur Geschichte der Universität Wien; Bd. VI), Graz-Köln 1965, pp. 87-88.

${ }^{6}$ Secondo la «Krasenlehre» di Carl Rokitansky (1804-78), allora dominante in Vienna.

${ }^{7}$ Riassunte nella mia comunicazione all'Istituto Lombardo di Scienze e Lettere il 10 Novembre 1983.

${ }^{8}$ Benjamin Travers, A case of aneurism by anastomosis in the orbit, cured by the ligature of the common carotid artery, in «Medico-chirurgical Transactions», 2 (1811), pp. 1-16 e Plate I.

${ }^{9}$ William Dalrymple, $A$ case of aneurism by anastomosis in the left orbit, cured by tying the common trunk of the left carotid, ibidem, 6 (1815), pp.111-123.

${ }^{10}$ Carl Hubert Sattler, Pulsierender Exophthalmus, in A.Graefe u. Th.Saemisch [edit.], Handbuch der gesamten Augenheilkunde (2.Aufl.), II.Teil, Kapitel XIII, Band IX, 1. Abt., 2. Teil, Berlin 1920, pp.1-268. Consulta nello stesso Handbuch anche: A. Birch-Hirschfeld, Der intermittirende Exophthalmus, Kap. XIII, 8, §§ 56-149, pp. 105-149.

${ }^{11}$ Walter Eduard Dandy, Carotid-cavernous aneurysms. (Pulsating exophthalmos), in «Zentralblatt für Neurochirurgie», 2 (1937), pp. 77-113 e 165-206.

12 Wallace B. Hamby, Carotid-cavernous fistula, Springfield, Illinois, 1966.

${ }^{13}$ Emile Delens, De la communication de la carotide interne et du sinus caverneux, Thèse de Paris 1870. La tesi è ornata di due perspicue tavole rappresentanti la fistola della carotide nel seno cavernoso. Una tavola è riprodotta a colori da Sattler (Fig. 19 a p.115). Entrambe le tavole sono inoltre riprodotte in bianco e nero nella monografia di Hamby (Fig. 1 a p. 10).

${ }^{14}$ Gunther Martin, Der Heldenberg. Führer durch die Gedenkstätte für Feldmarschall Radetzky in Klein-Wetzdorf, Niederösterreich, s.l.n. a.

\section{Riassunto}

Attorno al 1840 il Feldmaresciallo Radetzky soffrì gravemente per un «tumore» endorbitario, al quale il medico curante, l'omeopatico Hartung, attribuì carattere di malignità. Poco dopo che tale prognosi nettamente infausta era stata confermata da due professori di oculistica (Flarer in Pavia, Jäger in Vienna) il tumore cominciò a regredire fino alla guarigione completa nel corso di pochi mesi. Il successo fu ovviamente attribuito alle cure omeopatiche praticate da Hartung. L'autore propone, invece, la diagnosi di una fistola carotido-cavernosa, evoluta spontaneamente in guarigione. 


\section{Zusammenfassung}

Um das Jahr 1840 litt der Feldmarschall Radetzky sehr schwer an einer endorbitalen «Geschwulst», die von seinem Arzte, dem Homöopathen Hartung, als bösartig ausgelegt wurde. Kurz nachdem diese ausgesprochen verhängnisvolle Prognose von zwei Professoren der Augenheilkunde (Flarer in Pavia, Jäger in Wien) bestätigt worden war, begann die Geschwulst nach und nach zurückzugehen, bis zu einer vollständigen Heilung im Laufe von wenigen Monaten. Der Erfolg wurde natürlich der von Hartung angewandten homöopathischen Behandlung zugeschrieben. Der Verfasser neigt hingegen dazu, die Diagnose einer spontan ausgeheilten Karotisfistel im Sinus cavernosus zu stellen.

\section{Summary}

Around 1840, Fieldmarshall Radetzki suffered from a severe «tumor» in the orbita, which his physician, the homoeopath Hartung, diagnosed as malignous. Shortly after this diagnosis was confirmed by two professors of ophtalmology (Flarer in Pavia and Jaeger in Vienna), the tumor began to recede until complete disappearance within a few months. The success was naturally attributed to the homoeopathic cure. The author is, however, of the opinion, that the correct diagnosis would have been that of a spontaneously healing fistula of the carotis in the sinus cavernosus.

Prof. Dr.med. Luigi Belloni

Istituto di Storia della Medicina

Università degli Studi

Via Festa del Perdono, 7

I-20122 Milano 